\title{
EL CONSUMO EDITORIAL. HÁBITO DE LECTURA EN UNIVERSITARIOS DEL CENTRO UNIVERSITARIO DE CIENCIAS ECONÓMICO ADMINISTRATIVAS DE LA UNIVERSIDAD DE GUADALAJARA
}

\author{
Pedro Aguilar Pérez \\ Lucila Patricia Cruz Covarrubias \\ Pedro Daniel Aguilar Cruz \\ Universidad de Guadalajara (México)
}

RESUMEN: El estudio presenta una reflexión acerca de la situación existente en alumnos universitarios en la Universidad de Guadalajara, con relación a la práctica de leer y consumir productos editoriales. Se estudia la preferencia que tienen los estudiantes hacia las revistas, el diario y el libro. Desde ahí se teje la relación establecida entre consumo y lectura; para con ello entender la situación actual del consumo editorial en la U. de G. En el presente trabajo el tipo de investigación fue descriptiva, con diseño de campo, no experimental, para lo cual se elaboró y aplicó un instrumento de recolección de datos sobre hábito, consumo y preferencia de productos culturales, a una muestra donde intervinieron 491 estudiantes universitarios del Centro Universitario de Ciencias Económico Administrativas de la Universidad de Guadalajara. Los resultados indicaron que el hábito de la lectura no es muy predominante frente a otras actividades de ocio. En cuanto a la predilección del producto editorial se demostró que las revistas y periódicos son más favorecidas que el libro, en su consumo por los alumnos, y que el precio de éste si es un factor determinante en su adquisición. Se concluye que en cuanto al tiempo que se dedica a la lectura de un libro profesional no es muy alentador.

PALABRAS CLAVE: Consumo editorial, lectura, preferencia, hábito de lectura, libro.

\section{THE PUBLISHING CONSUMPTION. THE READING HABIT OF COLLEGE STUDENTS CUCEA UDG}

ABSTRACT: This paper presents a reflection on the situation in college students at the Universidad de Guadalajara, in relation to the practice of reading 
and consumption of publishing products. It examines the reading preferences of students to the magazines, newspaper and book. From there it weaves the established relationship between consumption and reading, to thereby understand the current state of consumer publishing at the $U$. de G. In this paper the type of research was descriptive, with field design, not experimental, for which it was developed and implemented an instrument to collect data on smoking, consumption and preference of cultural products, a sample which involved 491 college students Centro Universitario de Ciencias Económico Administrativas de la Universidad de Guadalajara. The results indicated that the habit of reading is not predominant over other leisure activities. As for the preference of the editorial product showed that magazines and newspapers are more favored than the book, in its consumption by students, and that the price of it if it is a factor in their purchase. We conclude that in the time devoted to reading a professional book is not very encouraging.

KEYWORDS: Consumer publishing, reading, preference, habit of reading a book.

Recibido: 18/05/2012

Aceptado: 27/09/2013

\section{INTRODUCCIÓN}

Según los resultados de investigaciones de varios autores, podemos decir que en nuestro país no existe la práctica de leer, mucho menos el hábito de la lectura ${ }^{1}$; hay quienes saben leer y escribir pero ni siquiera hojean un libro.

México cuenta con más de 112 millones de habitantes y alrededor de 1.800 librerías. ${ }^{2} \mathrm{Al}$ menos 18 millones de personas son lectores potenciales, pero el resto de la población no lee, no compra libros, no asiste con frecuencia a una biblioteca, son ciudadanos indiferentes a la literatura de calidad, mexicanos que se inclinan por la revista del momento, la del escándalo, la de chisme, o la novela policíaca, la vaquera, la pasional, literatura barata y popular, que vende y divierte.

La delimitación del sector editorial supone un punto de partida necesario para poder abordar en profundidad el estudio del consumo de productos editoriales en estudiantes universitarios. Con tal propósito consideramos obligado analizar y describir brevemente la estructura del mercado actual existente de la industria editorial en nuestro país.

En los años recientes la industria editorial mexicana se ha visto afectada por una saturación de productos editoriales en el mercado, que a su vez ha condicionado el crecimiento de las editoriales. La industria del sector editorial "que involucra producción de papel, talleres de impresión y encuadernación, editores, distribuidores y librerías" se ha deteriorado sensiblemente en la última década a causa del descenso

1. Para constatar la falta de hábito de lectura de libros y el estado mísero que presenta esta práctica, véase: Bartra, 1998; Chávez, 2002, González y Chávez, 1996; Muñoz, 1995; Ramírez, 2002; Zaid, 1996.

2. Según datos de la Cámara Nacional de la Industria Editorial Mexicana (2010). Disponible en: www. caniem.com. 
en la venta de libros y por el cierre de librerías. Pero no así en la creciente producción, edición, y venta de revistas y periódicos nacionales y extranjeros.

Las empresas editoriales lanzan al mercado gran cantidad de productos editoriales comerciales, lo vendible, Best Seller ${ }^{3}$, los lectores adquieren para leer lo que los editores producen. La sociedad dirige su interés hacia productos de mala calidad, como lo es el caso de las revistas de espectáculos ${ }^{4}$, por ejemplo, género de la industria editorial de entretenimiento que mantiene en acción a la práctica de la lectura masiva en México. ${ }^{5}$

Ante tal panorama, el objetivo de este trabajo, radica principalmente, en conocer la situación en la que se encuentra la práctica del consumo y hábito de la lectura en los alumnos de la Universidad de Guadalajara, específicamente en estudiantes de nivel superior en el área de las ciencias económico administrativas, para poder con ello identificar la lectura que mayormente prefieren y adquieren los universitarios y que le dan sentido al hábito de lectura.

Es así como se examinan unos de los problemas centrales del quehacer educativo actual: la compra de libros, revistas y periódicos y por consecuente la lectura y sus efectos para formar alumnos y profesionistas con un pensamiento crítico y reflexivo.

\section{EstruCtURA DEL MERCADO DE LA INDUSTRIA EDITORIAL}

La industria editorial en el sector del libro enfrenta en la actualidad varios problemas. La comercialización del libro es muy compleja. Desde el proceso creativo hasta la venta al público intervienen varios factores: el elevado número de novedades publicadas, el apoyo a los creadores, la edición universitaria, el precio de venta, la baja cantidad de librerías y la desproporción entre los espacios de exhibición y la producción editorial, el asunto del libro de texto, ${ }^{6}$ las bibliotecas de aula, los programas de apoyo y promoción de lectura.

En ese sentido el problema más importante que enfrenta la industria editorial, es la falta de lectores. Se estima que en México se leen 0.5 libros por persona y año, si no se tienen en cuenta los libros de texto (Rodríguez, 2000). Esta falta de lectores afecta al precio del producto ya que al ser las tiradas de las editoriales más pequeñas, es más costosa cada unidad producida. ${ }^{7}$

3. Expresión inglesa ("mejor vendido") con la que en la década de los años veinte de este siglo comenzó a denominarse al libro que, en determinado periodo de tiempo, había conseguido una mayor venta y difusión nacional o internacional. Los sociólogos que investigan el hecho de la difusión de la cultura destacan la complejidad y relatividad de libro concreto. Puede haber razones lingüísticas (una obra en inglés tiene más difusión, por contar con un mercado más amplio de hablantes y conocedores de ese idioma), económicas y culturales: alto nivel de vida y alfabetización del público al que se dirige.

4. Sólo basta con observar que las cinco revistas semanales con la más alta circulación son: Libro Semanal y Libro Vaquero con 41.6 millones de ejemplares vendidos por año, cada una, Libro Policiaco 28.6 millones, TV Y Novelas 28 millones y TV Notas con 21.8 millones. (Zacarías, 2002).

5. Véase, La Lectura Masiva en México: Apuntes y Reflexiones Sobre la Situación que Presenta Esta Práctica Social, en: Estudios sobre las Culturas Contemporáneas, año/vol. XI, número 021, Universidad de Colima, México, junio, pp. 71-84.

6. Según datos de la CANIEM, el Estado se convierte en el principal competidor de los editores mexicanos, al producir más del $60 \%$ de producción editorial mexicana.

7. La CANIEM informa que entre 1995 y 2006 el precio relativo de la clase libros y similares se incre- 
El sector editorial privado mexicano ha estado en la última década, disminuyendo su producción y su presencia internacional. ${ }^{8}$ En un informe de 2010 de la Cámara Nacional de la Industria Editorial Mexicana revela que en 2009 se publicaron 18.618 títulos, de estos el 33\% fueron novedades, mientras que en el 2007, fueron 20.300 títulos, esta proporción muestra una disminución en el número de títulos publicados.

En cuanto a la producción mexicana del sector privado en 2009 fue de 121.8 millones de libros y dentro de esa producción, 33.7 millones correspondió a la producción de libros para programas de gobierno (bibliotecas de aula y escolares y Educación secundaria). Mientras que en el año 2006 la producción fue de 137.5 millones de libros y de estos 54.1 millones fueron para los programas de gobierno (Ver tabla 1). La tendencia de la producción se muestra en decremento en los últimos tres años de $2.6 \%$. En cuanto a las ediciones propias, como en las importaciones, la tendencia es a la baja en 2009, y hubo decrementos de $7.3 \%$ en las ediciones propias y $5.1 \%$ en las importaciones, en comparación al 2007.

Tabla 1. La producción editorial del sector privado (millones)

\begin{tabular}{|l|c|c|c|c|}
\hline SECTOR EDITORIAL PRIVADO & Año 2006 & Año 2007 & Año 2008 & Año 2009 \\
\hline Producción de títulos (ediciones propias) & 83.4 & 92.6 & 90.5 & 88.1 \\
\hline $\begin{array}{l}\text { Producción del Sector privado adquirida } \\
\text { por el gobierno }\end{array}$ & 54.1 & 36.7 & 34.8 & 33.7 \\
\hline $\begin{array}{l}\text { Total de ejemplares producidos (de edición } \\
\text { propia más coediciones) }\end{array}$ & $\mathbf{1 3 7 . 5}$ & $\mathbf{1 2 9 . 3}$ & $\mathbf{1 2 5 . 3}$ & $\mathbf{1 2 1 . 8}$ \\
\hline
\end{tabular}

Fuente: elaboración propia de los autores.

En este marco, la desfavorable evolución de la ventas ha ocasionado que en la última década alrededor de $40 \%$ de los establecimientos de libros hayan cerrado, según informes de la Asociación de Libreros Mexicanos, A.C. (ALMAC).

Por otro lado, con datos de la CANIEM de 2007, en México existe una librería por cada 85.000 habitantes. Hay en el país 1.251 librerías y puntos de venta de libros

mentó $3.9 \%$ por año. Las ediciones científicas y libros y otras ediciones especiales registraron el mayor aumento de precios relativos (12.9\%) en ese período, seguidas por las ediciones humanísticas $(8.1 \%)$ y Técnicas (5\%).

8. En nuestro país, con datos de 1996 en L'état du monde, se publicaron, 6 mil 183 libros (títulos), mientras que en España se publicaron, el mismo año, 46 mil 330 y en Estados Unidos 68 mil 175 [...] En México los tirajes para una población de 100 millones de habitantes son ridículos, incluso en los periódicos: los siete principales diarios mexicanos editan la misma cantidad de unidades que uno de los principales de Argentina (con menos habitantes que México), para no hablar de los grandes diarios en Estados Unidos o en los países más desarrollados de Europa occidental [...] con datos de 1996 y 1997 según el país, Canadá publicó un titulo de libro por cada mil 532 habitantes, Estados Unidos un título por cada 4 mil 19 habitantes. México: un titulo por cada 15 mil 494 habitantes. Bélgica, Francia, España, Dinamarca, Suecia, Reino Unido, Japón son países con producción de títulos de libros superior a uno por mil 700 habitantes. Se dirá que estamos comparando a México con países desarrollados. Veamos los siguientes datos: Costa Rica (uno por 3 mil 714), Argentina (uno por 3 mil 667), Uruguay (uno por 3 mil 521), Chile (uno por 6 mil), Nigeria (uno por 8 mil 98) (Rodríguez, 2003: 13). 
(tiendas departamentales y centros comerciales). Las entidades federativas con el mayor número de puntos de ventas son el Distrito Federal con 464, el Estado de México con 87, Puebla con 60, Nuevo León con 56, Veracruz con 55 y Jalisco con 52. De hecho, estas seis entidades concentran $62 \%$ de las librerías del país. En el 2007, la red más grande de librerías es la que opera $\mathrm{EDUCAL}^{9}$, empresa adscrita al Consejo Nacional para la Cultura y las Artes, que cuenta con 82 en todo el territorio Nacional.

El Centro Regional para el Fomento del Libro en América Latina y el Caribe (CERLELC) (2004), señala que estos puntos de venta de libros están ubicados principalmente en "centros y zonas residenciales urbanas". Por otra parte, establece que en México no se han desarrollado las librerías especializadas con excepción de las dedicadas a asuntos religiosos, esotéricos, jurídicos, científicos y médicos.

Con datos de la CANIEM, las ventas en librerías representan $28 \%$ del total del mercado. Le siguen la venta de las distribuidoras con $20.6 \%$ y las compras del gobierno principalmente de libros de texto con 19.8\%. El 9\% del mercado del libro corresponde a exportaciones.

Finalmente, el mercado de libros establecidos legalmente se enfrenta al "submercado", la piratería y el fotocopiado ilegal. Según el Centro Mexicano de Protección y Fomento de los Derechos de Autor (CEMPRO), en México se piratean alrededor de 10 millones de libros al año.

Por otro lado, con un marco muy diferente, está el sector de la revistas, ya que éste en los últimos años ha tenido un crecimiento favorable. Con datos de la CANIEM, el volumen de producción de revistas pasó de 449 millones en 2001 a 714 millones en 2006, es decir se incrementó 59\% en ese período. Las revistas de interés general incrementaron su tiraje en 79 millones de ejemplares, las revistas femeninas en 71.7 millones y las revistas para hombres en 57 millones. Sin embargo, en términos relativos el mayor incremento se produjo en las revistas juveniles (430\%) e infantiles (221\%). La producción de revistas por habitante aumentó $10.2 \%$ en promedio anual. De 3.4 revistas por persona que se produjeron en 1998, se pasó a 6.9 revistas en 2006, es decir, que se duplicó.

Siguiendo, con datos de la CANIEM, en 2004 había en México 450 empresas privadas dedicadas a la edición de publicaciones periódicas. Según el rango del tiraje, el $74.3 \%$ de las empresas se clasificaba como pequeña o micro. En un rango medio se situaron el $12.9 \%$; las demás empresas se catalogaron como grandes ${ }^{10}$ y macro. Un porcentaje muy alto de empresas $(80.4 \%)$ se especializaban sólo en la edición de revistas, mientras que un (19.6\%) se orientaban a la edición de revistas o en combinación con otro tipo de publicaciones.

9. EDUCAL fue establecida en 1982 como una sociedad anónima de capital variable, con el objetivo social de diseñar material didáctico que apoyara al Sistema Educativo Nacional. A partir de 1998 por mandato de la presidencia de CONACULTA, se le asigna la función de comercializar y distribuir por medio de su infraestructura, los diferentes productos que generan las instituciones (públicas y privadas) culturales del país.

10. Editorial Televisa es el grupo editorial en español más grande a nivel mundial y líder en producción, representación y distribución en Latinoamérica y en el mercado hispano de Estados Unidos. Con una circulación de más de 140 millones de copias en el año 2000, Grupo Televisa publica más de 40 títulos que se distribuyen en 18 países incluyendo a México. http://www.esmas.com/editorial/televisa. 
Según el Instituto Nacional de Estadística Geografía e Informática (INEGI), en 2001 la producción de revistas fue de 137.4 millones de ejemplares; para 2006 el tiraje se había reducido a un poco más de 76 millones. La caída de la producción se debió al menor tiraje de revistas de historietas, que pasó de representar el $70.7 \%$ del total al $37.3 \%$.

En lo concerniente a los diarios, en México suman 340 periódicos los registrados. Los rotativos que tienen mayor posicionamiento y participación en el mercado son porque se financian a través de los ingresos percibidos por la publicidad, principalmente instituciones financieras y empresas trasnacionales, así como por la confianza y credibilidad que tienen los lectores hacia su contenido editorial. El total de la circulación a nivel nacional, registrada por el Instituto Verificador de Medios A.C. y por la agencia de publicidad (VYASA), ${ }^{11}$ es de 10.183.819 de periódicos diarios.

La información anteriormente expuesta revela una preocupante tendencia a la comercialización de cierto tipo de publicaciones donde los libros, como vehículo de generación de conocimiento especializado y cultural, simplemente no puede competir, y por lo tanto éste no consigue llegar a manos de los lectores (estudiantes).

\section{El caso del consumo editorial en alumnos universitarios de las ÁREAS ECONÓMICO-ADMINISTRATIVAS DE LA UNIVERSIDAD DE GuadALAJARA}

Este documento presenta el resultado del levantamiento de indagaciones a alumnos universitarios del Centro Universitario de Ciencias Económico Administrativas (CUCEA), de la Universidad de Guadalajara. El propósito fue conocer la opinión que existe con referencia al consumo de productos editoriales que hay en el mercado editorial. Particularmente se centra en identificar la percepción y limitaciones de los estudiantes universitarios sobre el conocimiento, gusto y consumo de los productos editoriales. Por otra parte, se identificaron las tendencias más importantes que se observan en el mercado editorial.

En tal sentido la investigación realizada fue de carácter descriptivo con un diseño no experimental transversal. La población objeto de estudio estuvo conformada por un total de 491 alumnos de todos los semestres y licenciaturas que se ofertan en el CUCEA.

Para la investigación se utilizó la modalidad del cuestionario simple que consistió en una técnica estructurada que contiene un conjunto de reactivos y alternativas de respuestas; los cuales fueron respondidos de forma directa por los alumnos encuestados. El instrumento elaborado, constituido por doce preguntas cerradas, se realizó en el mes de septiembre de 2010. El cuestionario está constituido por dos partes: una parte determina el hábito de la lectura y la preferencia de esta frente a otras actividades culturales, y la segunda parte se refiere al consumo del producto editorial.

Los datos se registraron en primer lugar bajo frecuencias absolutas, luego de contadas las opciones seleccionadas por lo sujetos, a quienes se les aplicó el instrumento que contenía sus respuestas, se transformaron en porcentajes de frecuencias relativas presentadas a través de Diagramas de barras y tablas.

11. Empresas que tienen por objetivo el promover el desarrollo de la actividad de la comunicación y auditar la circulación de medios en México. 


\section{ANÁlisis E INTERPRETACIÓN DE LOS RESUltados}

A continuación se presenta el análisis e interpretación de los resultados referidos a las opiniones expresadas por la población estudiantil estudiada, considerando los datos obtenidos, los objetivos planteados, secciones e indicadores.

En cuanto a los resultados obtenidos en el eje sobre el hábito de lectura se destaca que más del $50 \%$ de los alumnos encuestados afirmó leer más de una vez a la semana (el 55.8\%). Estos son quienes podrían denominarse como "lectores frecuentes". Por otra parte, existe un $22.8 \%$ que podríamos Ilamar "lectores esporádicos" ya que leen una vez a la semana. Del resto, existe más de $22 \%$ que se podría interpretarse que no tienen el hábito de leer, ya que leen de 2 a 3 veces por mes o una sola vez al mes.

En cuanto a la preferencia de leer frente a otras actividades de entretenimiento, se obtuvieron los siguientes resultados: la gráfica $\mathrm{n}^{\circ} 1$, muestra que el indicador que obtuvo el mayor porcentaje fue la televisión ya que un $46 \%$ de los alumnos encuestados la prefiere. Le sigue el indicador de leer, ya que este obtuvo una preferencia de un $19 \%$ de los entrevistados. Llama mucho la atención que solo un $1 \%$ prefiere ir a la librería, esto se puede interpretar que la población que prefiere leer, no precisamente opta por libros, sino otra clase de producto editorial (revistas y periódicos). Por último, el mayor porcentaje de los estudiantes prefiere otro tipo de producto cultural al producto editorial. Ver la televisión es lo que más hacen los alumnos en su rato de ocio.

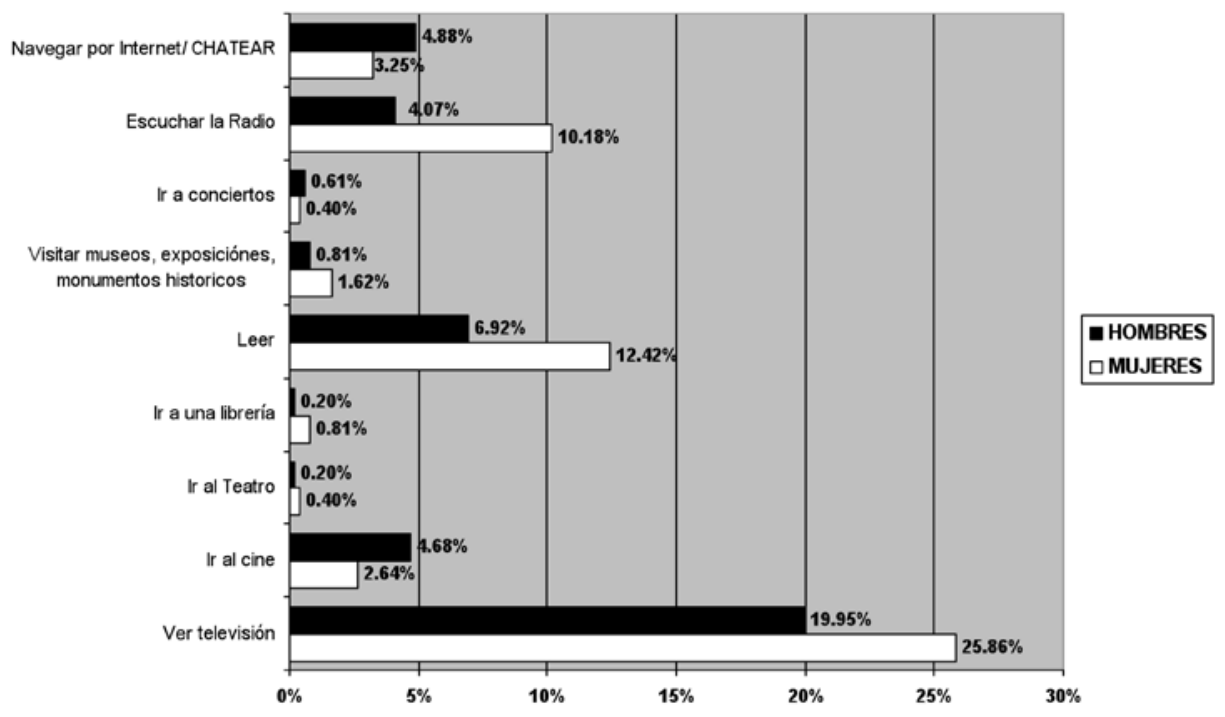

Gráfica $\mathbf{n}^{\mathbf{0}}$ 1. ¿ De las siguientes actividades cual realizas más seguido en tu tiempo libre? Fuente: Aguilar-Cruz (2010, con base en la encuesta realizada).

Es interesante observar la gráfica 1, ya que muestra el tipo de producto cultural que el alumno universitario demanda y prefiere consumir. Más de 220 alumnos prefieren ver televisión y solo 3 asisten al teatro. Pero también hay que tomar en cuenta 
que existen factores como educación, publicidad, ambiente social y en algunos casos el contenido y la calidad del producto cultural para que los jóvenes universitarios tomen su decisión.

En cuanto al género de lectura que prefieren los alumnos, el resultado (gráfica ${ }^{\circ}$ 2) arrojó lo siguiente: más del 25\% prefiere la lectura profesional, le sigue el 23\% en cuentos y revistas de predilección y un porcentaje de 16\% opta leer novela romántica, el resto 36\% elige leer libros de historia, de ficción, policíaco, de arte y teatro, cómico y otros. Aquí podemos observar cómo la mayoría de los estudiantes dedican su tiempo de lectura a los libros profesionales, cuentos y revistas, novelas románticas y libros de historia. También es importante señalar cómo las alumnas (mujeres) tienen preferencia en la lectura de cuentos, revistas y novelas en un gran porcentaje $(28.7 \%)$, a diferencia de los alumnos (hombres) con un $10.9 \%$.

口MUJERES $\approx$ HOMBRES

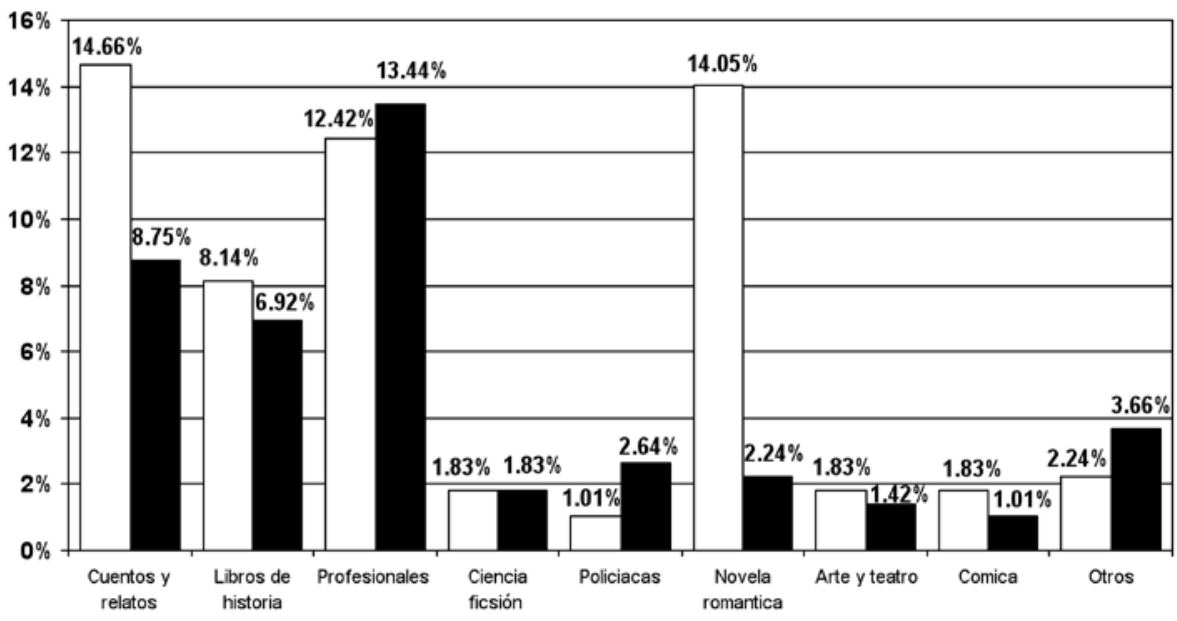

Gráfica no 2. ¿Qué género de lectura prefiere usted?. Fuente: Aguilar-Cruz (2010, con base en la encuesta realizada)

En el eje sobre el consumo del producto que se lee, la investigación arrojó los siguientes resultados. Con respecto, a qué clase de lectura ha comprado durante el último mes, más de $32 \%$ de los alumnos entrevistados señaló que compró libro profesional, un 26\% compró el periódico, le siguen las revistas ya que un 13\% compró éstas en el último mes y el resto de los encuestados más del 28\% compró cuentos, libros de ciencia ficción, arte y teatro, poesía y de superación.

En cuanto a ¿qué género de lectura compra más seguido?, la gráfica $n^{\circ} 3$ nos muestra que el $31.5 \%$ de los alumnos compra más seguido periódicos, los que compran libros continuamente son solo un $19.7 \%$ de los encuestados, le siguen en menor porcentaje los que compran revistas con un $15.8 \%$, con mucho menor porcentaje le siguen el género de romanticismo y/o poesía con un $7.1 \%$ y el de los cuentos con un $5.2 \%$ de los consultados. Es importante resaltar que las mujeres prefieren las revistas 
y cuentos más que los hombres, así como también éstos prefieren comprar más periódico que ellas. Con los resultados se puede interpretar que los encuestados prefieren comprar periódicos, revistas y cuentos, en lugar de un libro profesional.

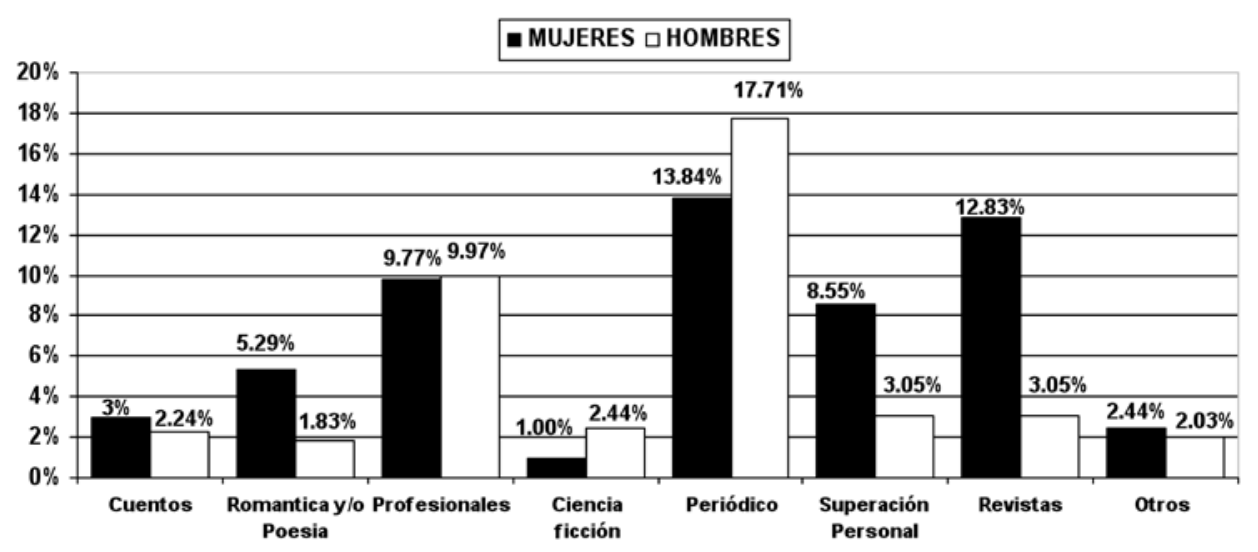

Gráfica no 3. ¿Qué genero de lectura compra usted más seguido? Fuente: AguilarCruz (2010, con base en la encuesta realizada)

Concerniente a este género de compra, se les preguntó a los alumnos encuestados ¿Qué libros compró en los últimos doce meses que no estén relacionados con su profesión o estudio? el 54\% manifestó que compró libros de superación personal, 18\% libros de ciencia ficción y el resto no compró libros de ningún género.

Con respecto a cuál es la forma como adquieren lo que leen, el 55.8\% de los encuestados señaló que lo que lee lo compra en una librería, le sigue el $25.6 \%$ quien expresó que lo obtiene por medio de préstamo ya sea de amigo(a) o familiar, un $6.1 \%$ comentó que es a través de regalo, con un $4 \%$ de los entrevistados señala que los adquiere por préstamo de la biblioteca de la escuela, con un porcentaje de más de $3 \%$ adquiere lo que lee porque lo fotocopia o los baja de Internet, y tan solo un $2.8 \%$ asiste a las bibliotecas públicas a que le presten lo que lee. Es importante señalar que la adquisición de lo que leen los entrevistados se refiere exclusivamente a los libros y no a otro género de lectura. También es trascendental marcar como ambos sexos por igual (más de 3\%) fotocopian o bajan de Internet lo que leen actualmente.

Sobre si el factor precio es una condicionante para adquirir un libro, el 66\% manifestó que sí, y solo el 34\% señalo que no es un factor determinante el precio. Por lo que se puede interpretar este resultado, que la mayoría de las personas no compra un libro por el precio que estos poseen.

En el caso de cuánto se estaría dispuesto a gastar por la adquisición de un libro, se puede observar en la Tabla 2 que el mayor número de universitarios encuestados optó por el rango de \$100 a \$200, independientemente del tipo de lectura del libro (excepto el del libro profesional donde más de $60 \%$ estaría 
dispuesto a gastar más de 200 pesos). Le sigue en preferencia el de \$201 a 300 pesos, luego el de \$301 a 400 pesos y con una distinción mínima los interrogados eligieron el de $\$ 401$ a 500 pesos, que estarían dispuestos a gastar en la compra de un libro. Cabe destacar que el indicador de libro académico (profesional) es el que tiene un porcentaje más equilibrado en todas sus secciones de precio, en comparación con los demás tipos de lectura de los libros. Estos resultados revelan que un alto porcentaje de los estudiantes están dispuestos a pagar no más de 200 pesos por adquirir un libro.

Tabla 2. ¿Cuánto estarías dispuesto a gastar por la adquisición de un libro?

\begin{tabular}{|l|c|c|c|c|}
\hline Tipo & $\mathbf{\$ 1 0 0}$ a 200 & $\mathbf{\$ 2 0 1}$ a 300 & $\mathbf{\$ 3 0 1}$ a $\mathbf{4 0 0}$ & $\mathbf{\$ 4 0 1}$ a 500 \\
\hline Cuento & $92 \%$ & $6.3 \%$ & $0.6 \%$ & $1.1 \%$ \\
\hline Romántica o poesía & $74.3 \%$ & $20.1 \%$ & $4.5 \%$ & $1.1 \%$ \\
\hline Cultural & $52.5 \%$ & $31.4 \%$ & $10.6 \%$ & $5.5 \%$ \\
\hline Académico (profesional) & $35 \%$ & $26.5 \%$ & $20.4 \%$ & $18.1 \%$ \\
\hline Ciencia ficción & $75.5 \%$ & $17.5 \%$ & $4.3 \%$ & $2.7 \%$ \\
\hline Superación Personal & $57.1 \%$ & $21.8 \%$ & $11.8 \%$ & $9.3 \%$ \\
\hline Técnico & $51.7 \%$ & $24.4 \%$ & $12.7 \%$ & $11.2 \%$ \\
\hline
\end{tabular}

Fuente: Aguilar-Cruz (2010, con base en la encuesta realizada).

Con respecto a la tabla anterior se podría interpretar que los estudiantes quieren comprar libros, pero a bajo costo. Por tal motivo los resultados arrojados demostraron que efectivamente el factor precio es una condicionante para la adquisición de un libro. De lo planteado anteriormente se puede inferir que los lectores (estudiantes universitarios) no compran libros y prefieren adquirirlos por otro medio o fotocopiarlos.

Sobre la pregunta que se efectuó a los encuestados de cuánto dinero gastan en la compra de algún genero de lectura, en un mes normal, se puede observar en la tabla 3 que la mayoría de los estudiantes no gasta mucho en adquirir algún tipo de género de lectura, solo los indicadores de prensa y libros profesionales (académicos, técnicos y culturales) y de ciencia ficción son excepciones, en los demás indicadores solamente gastan $\$ 60.00$ o menos en adquirir productos editoriales. Son muy interesantes los resultados mostrados en esta tabla, ya que nos revelan que en el indicador de la prensa una de cada tres personas está dispuesta a gastar en la compra de periódicos. Por su parte el indicador de las revistas deja ver que más del $42 \%$ sí gasta en la adquisición de éstas, así también es importante lo que revela el indicador en cuanto a libros académicos donde más del 47\% gasta más de 100 pesos al mes en adquirir un libro. En tal sentido los resultados de la Tabla 2 y 3 denotan que el mayor porcentaje de los lectores el factor precio es determinante en la adquisición de un texto, revista o periódico, resultados que deben ser observados muy de cerca a fin de que se tome en consideración por parte de todos los involucrados en la industria editorial, como el precio, es determinante en la compra del libro y esto repercute en el hábito de la lectura. 
Tabla 3. En un mes normal ¿cuánto dinero gastas en los rubros que se te especifican?

\begin{tabular}{|l|c|c|c|c|c|}
\hline \multicolumn{1}{|c|}{ Tipo } & $\begin{array}{c}\mathbf{\$ 2 0 0} \text { a } \\
\text { más }\end{array}$ & $\begin{array}{c}\mathbf{\$ 1 0 0} \mathbf{2 0 0} \\
\mathbf{2 0 0}\end{array}$ & $\begin{array}{c}\mathbf{\$ 6 0} \text { a } \\
\mathbf{3 0}\end{array}$ & $\begin{array}{c}\text { Menos } \\
\mathbf{d e ~} \mathbf{3 0}\end{array}$ & No gasto \\
\hline $\begin{array}{l}\text { Cuentos de relatos (lágrimas y risas, } \\
\text { memín pinguen etc.) }\end{array}$ & 11 & 23 & 21 & 51 & 385 \\
\hline $\begin{array}{l}\text { Prensa (mural, publico, financiero, } \\
\text { etc.) }\end{array}$ & 28 & 49 & 73 & 174 & 167 \\
\hline $\begin{array}{l}\text { Revistas (TV Notas, TV y Noveles, } \\
\text { Vanidades, Cosmopolitan, etc.) }\end{array}$ & 9 & 22 & 52 & 125 & 283 \\
\hline $\begin{array}{l}\text { Libros académicos, técnicos y } \\
\text { culturales. }\end{array}$ & 109 & 121 & 46 & 23 & 192 \\
\hline $\begin{array}{l}\text { Novelas policíaca, terror, cómica, } \\
\text { etc. }\end{array}$ & 14 & 42 & 26 & 38 & 371 \\
\hline $\begin{array}{l}\text { Libro de Ciencia ficción (Harry } \\
\text { Potter, El señor de los anillos, } \\
\text { Narnia, etc.) }\end{array}$ & 34 & 51 & 15 & 29 & 362 \\
\hline
\end{tabular}

Fuente: Aguilar-Cruz (2010, con base en la encuesta realizada).

\section{Conclusiones}

De cara a futuras investigaciones, detectamos algunos aspectos a los que convendría prestar más atención. Entre ellos, la necesidad de incluir una muestra más específica de mujeres y hombres, para explorar posibles efectos asociados al género, que con nuestros datos no se pudieron conocer. También sería necesario conocer más aspectos relacionados con hábitos de lectura y su relación con la formación académica del estudiante, para ello sería deseable utilizar medidas más precisas (diseños cualitativos y cuantitativos) de los hábitos culturales y factores motivacionales que propicia la universidad sobre su preferencia lectora.

Del análisis de los datos recabados en la investigación se generan las siguientes conclusiones:

- En cuanto al hábito de la lectura. Los resultados proporcionaron una información valiosa (para los maestros, autoridades educativas y empresas editoriales), en materia del hábito de leer, efectivamente, a los estudiantes sí les gusta la lectura, aunque no precisamente son libros lo que consumen y prefieren, sino lo contrario, literatura barata, como son muchas revistas y cuentos (de espectáculos y de entretenimiento) que se encuentran en el mercado. Lo que evidencia que el mercado editorial enfrenta dos retos fundamentales: desarrollar un mercado de lectura, de manera sostenida, para lo que se requiere el concurso de muchos agentes -gobierno, empresarios, sistema educativo, docentes, bibliotecas, padres de familia, etc. - y desarrollar una oferta adecuada para ese mercado. Este último tema toca a toda la cadena productiva del libro. Una oferta adecuada implica contar con empresarios avezados y con mayor interés al riesgo, que le apuesten a la aventura de hacer leer a los mexicanos, con más libros, de mejor calidad y a precios competitivos. 
- Se tienen que desarrollar otros canales que permitan llegar a más personas y a la formación de nuevos lectores, a través de medios de comunicación, como la radio, televisión e internet, así como foros de debate y noticias en la prensa, con el propósito de llamar la atención de los estudiantes hacia la lectura especializada y de calidad.

- En cuanto al consumo del producto editorial, el resultado demuestra que uno de los factores que más intervienen en el consumo de productos editoriales, es el precio, y que este es un elemento fundamental para la aceptación o no de la adquisición de un libro de buena calidad en su contexto. Por ello, es de fundamental importancia, que las políticas que se diseñen a favor de la Industria Editorial no se limiten a apoyar solamente la producción, sino que, pongan especial énfasis en la difusión y distribución de los productos editoriales, ya que estas áreas intervienen en el precio final del libro y por lo tanto en su consumo. Así como la edición de nuevas colecciones de libros de circulación masiva, precios bajos y publicación de ciertos materiales en medios electrónicos. Esto con el fin de que los estudiantes de niveles medios y superiores tengan la oportunidad de adquirir productos editoriales (libros y revistas) de calidad, para su desarrollo profesional, personal y cultural.

Desde una perspectiva en el ámbito educativo, es necesario reflexionar sobre el lugar que adquieren las prácticas de lectura en el ámbito académico del Nivel Superior. Se necesitan acciones o proyectos institucionales, en donde la Universidad pueda brindar y fortalecer los caminos hacia la lectura de libros científicos, con políticas que emerjan desde los currículos de manera eficiente. Creemos que es necesario desarrollar un diagnostico sobre el número de libros-textos que leen los estudiantes, una cualificación disciplinar de ellos y un diseño de un plan programático para cada carrera.

A nivel universitario, el docente, se ubica como el agente dinamizador más directo y mediador de la lectura en la Universidad, por ello su protagonismo en la práctica, hábito y consumo de la lectura debe ser ingente. En consecuencia el profesor debe potenciar la clase, motivar y promover las competencias lectoras desde el aula, para ello y en función de los conocimientos, deberá contar con las habilidades, aptitudes, actitudes y valores para el desempeño de su quehacer pedagógico, por lo que se debe crear un programa de capacitación para los docentes de la Universidad de Guadalajara, a través de talleres o seminarios de lectura textual, para que sean asesores y consultores para la comprensión de lecturas académicas. Atender los hábitos y preferencias de lectura de los universitarios, guiar a éstos en lecturas disciplinares, verificar en sus currículos el contexto de lectura en el que se mueve el estudiante y proyectar una interdisciplinaridad de lectura en los programas académicos con los demás profesores en tarea inminente de todo docente.

Es urgente y necesario que los profesores se actualicen en las nuevas formas del texto electrónico para estar en correspondencia con los avances y nuevas tecnologías que median entre los estudiantes y la cultura moderna.

Por su parte, el estudiante universitario, sujeto y objeto de esta investigación, tiene el compromiso de: ser su propio agente dinamizador, debe comenzar con 
una toma de conciencia sobre el hábito y consumo del producto editorial, que le proporciona la lectura de éste y con actitud, disciplina y práctica, desarrollar y perfeccionar su competencia lectora.

No podemos finalizar sin subrayar el interés práctico a la hora de diseñar procedimientos para estimular a los estudiantes a la lectura, no solo por la vía de que ésta puede representar de aumento del conocimiento, sino de posible herramienta para satisfacer algunos de los motivos básicos académicos. Cuestiones como el carácter hedónico de la lectura manifestado en términos de la preferencia por la literatura "barata", serían ejemplos de variables que no pueden pasar desapercibidas por las autoridades universitarias y los profesores y fomentar los hábitos de lectores de jóvenes universitarios de nuestro país.

Se concluye diciendo que todos tenemos que trabajar en dos planos, la entidad cultural y la entidad comercial. Que todos los involucrados en la promoción de la lectura, diseñen mecanismos adecuados mediante estrategias metodológicas y técnicas pedagógicas donde se motiven a los estudiantes a leer más y mejor, para contribuir a incrementar el hábito de la lectura de libros en nuestro país.

\section{REFERENCIAS BIBLIOGRÁFICAS}

ASOCIACIÓN DE LIBREROS MEXICANOS (2004). Informe Estadístico 2004. Disponible en: http://www.almac-nacional.org.mx.

BARTRA, R. (1998). Libros hambrientos, lectores escasos. Reforma, México, 10 de febrero (En sección: Cultura).

CÁMARA NACIONAL DE LA INDUSTRIA EDITORIAL MEXICANA. (2010). Informe Anual 2010. Disponible en: http://www.caniem.com.

CARRILLO, R. (2002). Pasado inmediato. ¿Hacia un país sin lectores? El Comentario, Colima - México.

CENTRO MEXICANO DE PROTECCIÓN Y FOMENTO DE LOS DERECHOS DE AUTOR. (2008). Informe Anual 2009. Disponible en: http://www.cempro.com.mx.

CHÁVEZ, G. (2002). Practica de la lectura en México y el libro como producto cultural. Colima: Universidad de Colima.

CHÁVEZ, G. (2005). La Lectura Masiva en México: Apuntes y Reflexiones Sobre la Situación que Presenta esta práctica social. Estudios sobre las Culturas Contemporáneas, XI (021), 71-84.

EDITORIAL TELEVISA (2007). Informe. Disponible en: http://www.esmas.com/ editorial/televisa.

GONZÁLEZ, J. A. y CHÁVEZ, M. G. (1996). La cultura en México I. Cifras clave. Colima: CNCA/Universidad de Colima.

GOODMAN, K. (1996). La lectura, le escritura y los textos escritos: una perspectiva transaccional sociopsicolingüística. Textos en contextos II. Lectura y Vida.

INSTITUTO NACIONAL DE ESTADÍSTICA GEOGRAFÍA E INFORMÁTICA (2011). Disponible en: http://www.inegi.gob.mx.

LERNER, D. (2001). Leer y escribir en la escuela. Lo real, lo posible y lo necesario. México: Fondo de Cultura Económica. 
MUÑOZ, S. (1995). El ojo, el libro y la pantalla. Consumo Cultural en Cali. Colombia: Facultad de Humanidades.

RAMíREZ, F. (2002). Al año leen mexicanos de 1.5 a 3 libros. El Financiero, México, 2 de octubre.

RODRÍGUEZ, X. (2000). Escritores y poder. La dualidad republicana en México 19681994. CONACULTA/FONCA/Universidad Pedagógica Nacional.

RODRíGUEZ, O. (2002). De autores y libros. La Jornada, México, 10 de enero (En sección: Política/opinión).

ZAID, G. (1996). Los demasiados libros. México: Océano.

ZACARÍAS, A. (2002). El Panorama del mundo editorial en el sector universitario. México: La Colección de Babel, Universidad de Guadalajara. 